\title{
Psychosocial Syndemic of suicidal ideation: a cross-sectional study among sexually transmitted infection patients in Shanghai, China
}

Suping Wang ${ }^{1+}$, Yang $\mathrm{Ni}^{2+}$, Ruijie Gong ${ }^{1,3+}$, Yuan Shi ${ }^{1}$, Yong Cai ${ }^{1 *}$ (b) and Jin Ma ${ }^{1 *}$

\begin{abstract}
Background: Patients with sexually transmitted infections (STIS) experience difficulties with stability and trust in long-term relationships and have poor mental health, factors that may lead to suicidal ideation. We sought to verify whether psychosocial health problems among patients with STIs were associated with these patients' suicidal ideation and to examine the syndemic effect of multiple psychosocial problems on suicidal ideation.

Methods: This was a cross-sectional study of 519 STI patients at the Shanghai Skin Disease Hospital. Demographic, psychosocial, and suicidal ideation information about the participants was collected by questionnaire. Logistic regressions were performed to detect the association between demographic variables and suicidal ideation, as well as each individual psychosocial variable and suicidal ideation, and to verify the syndemic effect of psychosocial factors.

Results: Of the participants, 25.0\% (130/519) reported having suicidal ideation. In univariable analysis, low self-esteem, loneliness, depression, entrapment, defeat, and unsatisfied interpersonal needs were associated with suicidal ideation. Multivariable analysis found depression (odds ratio [OR]: 4.1; 95\% confidence interval [Cl]: 2.3-7.2) and entrapment (OR: $2.1 ; 95 \% \mathrm{Cl}: 1.1-4.1)$ each had a more significant relation with suicidal ideation than the other psychosocial problems examined. STI patients who experienced two or more psychosocial health problems had approximately fourfold odds of suicide ideation (adjusted OR [AOR]: 4.2; 95\%Cl: 2.6-6.8) compared with those in the non-syndemic group, especially in the high-level (five or more psychosocial problems) group (AOR: 7.0; 95\%Cl: 3.9-12.5).

Conclusions: The study found the participants had a high rate of suicidal ideation and suffered from severe psychosocial problems. These results show a syndemic effect of psychosocial problems on increasing the odds of suicidal ideation. Our findings suggest an urgent need for efforts to prevent suicidal ideation among STI patients toward improving the social and health conditions of this population.
\end{abstract}

Keywords: Syndemic, Psychosocial problems, Suicidal ideation, Sexually transmitted infection patients

\footnotetext{
*Correspondence: caiyong202028@hotmail.com; majin@shsmu.edu.cn

${ }^{+}$Suping Wang, Yang Ni and Ruijie Gong contributed equally to this work.

'School of Public Health, Shanghai Jiao Tong University School of Medicine,

No. 227 South Chongqing Road, Shanghai 200025, China

Full list of author information is available at the end of the article
}

(c) The Author(s). 2020 Open Access This article is licensed under a Creative Commons Attribution 4.0 International License, which permits use, sharing, adaptation, distribution and reproduction in any medium or format, as long as you give appropriate credit to the original author(s) and the source, provide a link to the Creative Commons licence, and indicate if changes were made. The images or other third party material in this article are included in the article's Creative Commons licence, unless indicated otherwise in a credit line to the material. If material is not included in the article's Creative Commons licence and your intended use is not permitted by statutory regulation or exceeds the permitted use, you will need to obtain permission directly from the copyright holder. To view a copy of this licence, visit http://creativecommons.org/licenses/by/4.0/. The Creative Commons Public Domain Dedication waiver (http://creativecommons.org/publicdomain/zero/1.0/) applies to the data made available in this article, unless otherwise stated in a credit line to the data. 


\section{Background}

According to the World Health Organization, one person in the world commits suicide every $40 \mathrm{~s}$. However, suicide is preventable. Suicidal ideation, defined as thinking about, considering, or planning for suicide [1], may be a prodrome for ultimately committing suicide. Those with suicidal ideation have higher risk for suicide than those without. Suicidal ideation may also indicate a person suffers from great distress and psychological burden. Sexually transmitted infection (STI) patients experience difficulties with stability and trust in long-term relationships [2] and have poor mental health [3-7], factors that may lead to suicidal ideation.

One study in Canada found that $6.7 \%$ of STI patients had suicidal ideation and mental health needs over the previous 12 months [8]. Other investigations have examined people living with the human immunodeficiency virus/acquired immunodeficiency syndrome (HIV/AIDS; PLWHA), the range of suicidal ideation was 27.2 to $43.1 \%$ [9-12].

In one survey of STI patients in Jamaica, $65.5 \%$ of participants screened positive for at least one psychosocial problem, including depression and suicidal ideation [8]. Previous studies also revealed the association between certain psychosocial problems and suicidal ideation. Additionally, studies have confirmed that low self-esteem and depression are the principal factors behind suicidal ideation [13-23], and morecurrent articles have reported that loneliness, unsatisfied interpersonal needs, entrapment, defeat, and poor social support are also strong predictors $[17,19,24-$ 26]. One study in China reported poor social support as the strongest predictor of suicidal ideation, depression and low self-esteem were also strong predictors [24]. In two recent studies, one in the United States reported that loneliness and poor social support might represent the most important components of connectedness because they were found to be associated with depression severity and suicidality; the other study in Swaziland reported feeling lonely as a risk factor for suicidal ideation [27, 28]. Defeat and entrapment are key variables of Williams' cry of pain model of suicide and central to O'Connor's integrated motivational-volitional (IMV) model [29, 30]. Recently, Joiner proposed the interpersonal theory of suicide (IPTS) [31], which asserts that suicidal ideation emerges when individuals experience thwarted belongingness (loneliness and lack of reciprocal care) and perceived burdensomeness (perceived liability to others and self-hate).

Notably, individuals with several psychosocial problems may have magnified suicidal ideation $[9,32,33]$. We thus introduce the syndemic theory to this discussion. "Syndemic" as a term was first proposed by Singer to describe "synergistically related" epidemics that cluster and arise from harmful social conditions [34]. A syndemic effect, or synergistic epidemic, is the aggregation of two or more concurrent or sequential epidemics or disease clusters in a population. This effect exacerbates the disease prognosis and burden. To our knowledge, most research on the mechanism of suicidal ideation among STI patients (including PLWHA) has focused on a single psychosocial factor $[12,35]$. These studies also address behavioral habits, risky sexual behaviors, unpleasant sexual experiences, and psychosocial problems. There is inherent difficulty in obtaining truthful answers because such matters involve a high degree of privacy. In China's mainstream culture this issue is a particular challenge. Some research has even considered STIs as a factor behind the syndemic effect on suicidal ideation, whereas the present study focused particularly on STI patients' psychosocial problems [33, 34, 36]. Only one study has discussed the association between a syndemic effect of psychosocial complements (depression, selfesteem, and social support) and suicidal ideation among HIV+ patients in Nanjing, China [33]. Among other populations in China, our team identified a psychosocial syndemic effect (self-esteem, depression, social support, and loneliness) and suicidal ideation in men who have sex with men (MSM) [12]. The present study used defeat, entrapment, and poor interpersonal need as psychosocial factors, in addition to depression, low self-esteem, loneliness, and poor social support, to determine whether the syndemic effect would be similar among STI patients while considering the association with suicidal ideation.

In this study, we aimed to verify three hypotheses: [1] STI patients suffer several psychosocial problems and a high rate of suicidal ideation [2]; suicidal ideation is strongly associated with psychosocial problems; and [3] there is a psychosocial syndemic effect in suicidal ideation among STI patients.

\section{Methods \\ Participants}

This cross-sectional study, started in November 2017, was conducted for 6 months among STI clinic patients in two branch institutes of the Shanghai Skin Disease Hospital: Qiujiang Road and Baode Road. This hospital specializes in STIs and is one of the premier medical institutes in this field.

In accordance with the Law of the People's Republic of China on the Prevention and Treatment of Infectious Diseases, "STI patients" herein refers to those with any of five types of STIs that need preventive measures: syphilis, gonorrhea, genital warts, genital herpes, and HIV. All diagnoses (including clinical diagnoses and laboratory diagnosis) were conducted by sexually 
transmitted disease (STD) doctors at the hospital. Individuals who met the following criteria were invited to take part: $\geq 18$ years old, clinically diagnosed with an STI disease, able to read the informed consent form, and no participation in a similar study in the previous 6 months.

Patients who had any of the following criteria, were excluded: severe mental or cognitive impairment (e.g., neurosyphilis), unconsciousness, or disinclination to participate $(n=88)$.

Assuming $30.0 \%$ prevalence of lifetime suicidal ideation in STI patients, using alpha of 0.05 and a relative error for sampling of 0.15 , we calculated a required sample size of $415[7,33]$. To allow for a $30 \%$ non-response rate, a total of 540 patients were recruited to participate, and 519 (96.1\%) valid questionnaires were collected.

\section{Ethics}

The Shanghai Jiao Tong University School of Medicine Public Health and Nursing Ethics Committee approved the study (approval number: SJUPN-201702). Background information on the survey was given orally to all participants, after which they were given written informed consent forms that set out the study's goal, procedures, and potential risks. Participants signed informed consent forms before the study began. During the recruitment, participants were free to ask any questions and to withdraw.

\section{Procedure}

Our survey team signed cooperation agreements with the Shanghai Skin Disease Hospital before the investigation. All the doctors worked in the STD Department (inpatient or outpatient) were recruited and informed about the survey beforehand. All the investigators were students (seniors or postgraduates) from Shanghai Jiao Tong University School of Medicine. They received presurvey training and several in-person reviews throughout the study. The training also incorporated quality-control strategies, such as reexamining and investigating the questionnaires and resolving issues that may arise during the fieldwork.

The doctors informed each participant who met the inclusion criteria about the survey. After initial approval, participants were asked to enter a separate room to attend an interview conducted by our investigators. The interviews were divided into two parts: [1] Our investigators informed participants about the study goal, study procedure, and potential risks before participants signed the informed consent form [2]. The participants completed anonymous questionnaires in a separate room to protect their privacy and to confirm the validity of the questionnaire data. The investigators only provided assistance upon request.
Each participant received 80 RMB (approximately USD 12) cash for his/her participation.

Our investigators went to the two branch institutes of the Hospital every Wednesday and Saturday during the study period. Patients who visited one of the two branch institutes to see a doctor for STI treatment on a Wednesday and Saturday (when our investigators were present) were regarded as potential objects. Doctors at the institutes assessed whether patients met our eligibility criteria and inquired about participation. No appointment system was implemented at the two institutes, so our investigators meeting the patients was accidental. Thus, we employed accidental sampling.

\section{Measures}

\section{Demographic variables}

Demographic variables included age, sex, educational level, marital status, residency, income, insurance, sexual orientation, and HIV status.

\section{Suicidal ideation}

Suicidal ideation was measured via one question [12]: "Have you ever thought about committing suicide?" (hereinafter defined as suicidal ideation; $0=$ no, $1=$ yes).

\section{Psychosocial variables \\ Self-esteem}

Self-esteem was assessed based on the 10-item Rosenberg Self-Esteem Scale (SES) [37]. Negative statements such as "all in all, I am inclined to feel that I am a failure" required a reverse in score (e.g., $0=3,1=2,2=1$, $3=0$ ) before adding to the total. The Chinese version was used and low self-esteem was indicated with a result $<29$ (the norm for a Chinese population in China was 28.75 [38]) (Cronbach's $\alpha$ : 0.847; range: 12-40).

\section{Loneliness}

The initial version of the UCLA Loneliness Scale used 20 items (e.g., "I lack companionship") designed to estimate participants' loneliness and related emotional states [39]. Hays and DiMatteo identified a highly correlated alternative of eight items (eight-item UCLA Loneliness Scale: ULS-8), to achieve similar reliability but reduce the respondent's time burden and improve data quality [40]. In this study, we used the Chinese version of ULS-8 [41] (Cronbach's $\alpha$ : 0.820; range: 8-32). The more loneliness the individual felt, the higher that person would score, with a cutoff point set at the 75th percentile; a score of 18 .

\section{Depression}

Depression level was gauged using the Chinese version of Patient Health Questionnaire-9 (PHQ-9), a brief screening tool comprising nine items that match diagnosis criteria in the Diagnostic and Statistical Manual of 
Mental Disorders, Fourth Edition. Individuals were asked to recall how often they had experienced troubling problems (e.g., "little interest or pleasure in doing things") over the preceding 2 weeks, scoring from 1 (not at all) to 4 (nearly every day) [42]. A cutoff point of an algorithm score of 5 showed good screening performance within various settings [43] (Cronbach's $\alpha$ : 0.910; range: 0-27).

\section{Entrapment}

Evaluation of entrapment was quantified using the Chinese version of 16-item Entrapment Scale (ES) [44, 45], which reflected the escape motivation triggered either by perception of the outside world (e.g., "I am in a relationship I can't get out of") or internal feelings (e.g., "I want to get away from myself") (Cronbach's $\alpha$ : 0.965; range: $0-64)$. Higher than the 75th percentile-a score of 21was defined as high entrapment.

\section{Defeat}

For the 16-item Chinese version of Defeat Scale (DS) $[44,46]$, participants were asked about how they had thought about themselves in the preceding 7 days. Three items $[2,4,9]$ were recoded before computing. Their scores were calculated by summing the items for each scale (scored 0-4) (Cronbach's $\alpha$ : 0.886; range: 0-56). Higher than the 75th percentile-a score of 23-was defined as high defeat.

\section{Interpersonal needs}

Interpersonal needs were measured using the 15-item Chinese version of the Interpersonal Needs Questionnaire (INQ-15), which measures perceived burdensomeness (items 1 to 6) and thwarted belongingness (items 7 to 15) [47, 48]. Respondents rated how often they felt a certain way (e.g., "These days, the people in my life would be better off if I were gone"), scored from 1 (not at all true for me) to 7 (very true for me). Six items $[7,8,10,13,49]$ were recoded before computing. Higher than the 75 th percentile score of 49 was defined as interpersonal needs being unsatisfied (Cronbach's $\alpha$ : 0.855; range: 15-96).

\section{Perceived social support}

The Chinese version of the Multidimensional Scale of Perceived Social Support (MSPSS) is a 12-item, sevenpoint Likert scale based on the self-reported measure of support received from family, friends, and/or other people of special significance (e.g., "There is a special person with whom I can share joys and sorrows") [50]. Scoring ranged from 1 (very strongly disagree) to 7 (very strongly agree). A higher score indicated better social support, and the 25th percentile-a score of 59-was adopted as the cutoff point (Cronbach's $\alpha$ : 0.947 ; range: $12-84$ ).

\section{Psychosocial problems}

Psychosocial problems included low self-esteem, high loneliness, high depression, high entrapment, high defeat, unsatisfied interpersonal needs, and low perceived social support. Low self-esteem was defined as individuals who scored $\leq 29$ points on the SES. High loneliness was defined as those who scored $>18$ on the ULS-8. High depression was defined as individuals who scored $>5$ points on the PHQ-9. High entrapment was defined as those who scored $>21$ points on the ES. High defeat was defined as individuals who scored $>23$ points on the DS. Unsatisfied interpersonal need was defined any those who scored $>49$ points on the INQ-15. Low perceived social support was defined as individuals who scored $\leq 59$ points on the MSPSS.

\section{Syndemic effect of psychosocial variables}

Two or more concurrent psychosocial problems in a participant indicated a syndemic phenomenon [51]. The syndemic effect was established via the number of concurrent problems. Five or more indicated a high level of syndemic effect; otherwise, the participant had a low level.

\section{Statistical analysis}

Statistical analysis was performed using IBM SPSS Statistics for Windows, Version 22.0 (IBM Corp., Armonk, NY, USA). First, baseline descriptive statistics were calculated to summarize demographic characteristics, suicidal ideation, and psychosocial variables. Univariable analysis was then conducted via binary logistic regression to detect the association between demographic variables and suicidal ideation, and between univariable psychosocial variable and suicidal ideation. After adjusting for all significant demographic variables, univariate logistic regression analysis was performed to examine the psychosocial syndemic effect on suicidal ideation among these STI patients. Multivariable logistic regression was then used to evaluate all seven psychosocial problems associated with suicidal ideation, after adjusting for significant demographic variables. In the final stage, syndemic count variables were created by counting each individual's number of psychosocial health problems, and different groups were established based on the number of variables.

\section{Results}

\section{Demographic characteristics of participants}

In all, 519 patients (median age, 34.0 years) were investigated: $25.0 \%$ (130/519) had experienced suicidal ideation; 9.6\% (50/519) were HIV-positive; 47.6\% (247/519) were diagnosed with genital warts; and $23.9 \%$ (124/519) were diagnosed with syphilis (Table 1 ). 
Table 1 Demographic characteristics of the participants and associations with suicidal ideation $(N=519)$

\begin{tabular}{|c|c|c|c|c|c|}
\hline \multirow{2}{*}{$\begin{array}{l}\text { Demographic } \\
\text { Characteristics }\end{array}$} & \multicolumn{2}{|c|}{ Number of participants } & \multicolumn{3}{|c|}{ Had suicidal ideation } \\
\hline & $\mathrm{n} / \mathrm{N}$ & column(\%) & $n / N$ & $\operatorname{row}(\%)$ & $\mathrm{OR}(95 \% \mathrm{Cl})$ \\
\hline \multicolumn{6}{|l|}{ Case } \\
\hline Outpatient & $401 / 519$ & 77.3 & $98 / 401$ & 24.4 & $0.9(0.5-1.4)$ \\
\hline Inpatient & $118 / 519$ & 22.7 & $32 / 118$ & 27.1 & 1 \\
\hline \multicolumn{6}{|l|}{ Age group $^{a}$} \\
\hline$<25$ & $54 / 519$ & 10.4 & $25 / 54$ & 46.3 & $4.7(1.6-13.9)^{* *}$ \\
\hline $25-40$ & $326 / 519$ & 62.8 & $85 / 326$ & 26.1 & $1.9(0.7-5.2)$ \\
\hline $41-59$ & $107 / 519$ & 20.6 & $15 / 107$ & 14.0 & $0.9(0.3-2.6)$ \\
\hline$\geq 60$ & $32 / 519$ & 6.2 & $5 / 32$ & 15.6 & 1 \\
\hline \multicolumn{6}{|l|}{ Sex } \\
\hline Male & $232 / 519$ & 44.7 & $50 / 232$ & 21.6 & $0.7(0.5-1.1)$ \\
\hline Female & $287 / 519$ & 55.3 & $80 / 287$ & 27.9 & 1 \\
\hline \multicolumn{6}{|l|}{ Education $^{\text {b }}$} \\
\hline Middle School or less & $114 / 519$ & 22.0 & $38 / 114$ & 33.3 & $1.6(0.9-2.5)$ \\
\hline High School & $98 / 519$ & 18.9 & 18/98 & 18.4 & $0.7(0.4-1.3)$ \\
\hline College degree or above & $307 / 519$ & 59.1 & $74 / 307$ & 24.1 & 1 \\
\hline \multicolumn{6}{|l|}{ Current Marital Status } \\
\hline Married & $314 / 519$ & 60.5 & $63 / 314$ & 20.1 & $0.5(0.3-0.8)^{* *}$ \\
\hline Divorce & $32 / 519$ & 6.2 & $8 / 32$ & 25.0 & $0.7(0.3-1.6)$ \\
\hline Widowed & $10 / 519$ & 1.9 & $5 / 10$ & 50.0 & $2.0(0.6-7.3)$ \\
\hline Unmarried (Single) & $163 / 519$ & 31.4 & $54 / 163$ & 33.1 & 1 \\
\hline \multicolumn{6}{|l|}{ Income (RMB) ${ }^{c}$} \\
\hline$\geq 12,001$ & $123 / 519$ & 23.7 & $22 / 123$ & 17.9 & $0.4(0.2-0.8)^{* *}$ \\
\hline $6001-12,000$ & $168 / 519$ & 32.4 & $41 / 168$ & 24.4 & $0.6(0.3-1.1)$ \\
\hline $3001-6000$ & $155 / 519$ & 29.9 & $42 / 155$ & 27.1 & $0.7(0.4-1.3)$ \\
\hline$\leq 3000$ & $73 / 519$ & 14.0 & $25 / 73$ & 34.2 & 1 \\
\hline \multicolumn{6}{|l|}{ Residence Status $^{d}$} \\
\hline Local & $227 / 519$ & 43.7 & $41 / 227$ & 18.1 & $0.6(0.4-1.1)$ \\
\hline Stay less than 1 year & $85 / 519$ & 16.4 & 29/85 & 34.1 & $1.5(0.8-2.7)$ \\
\hline Stay $1-5$ year & $65 / 519$ & 12.5 & $24 / 65$ & 36.9 & $1.7(0.9-3.2)$ \\
\hline Stay more than 5 year & $142 / 519$ & 27.4 & $36 / 142$ & 25.4 & 1 \\
\hline \multicolumn{6}{|c|}{ Self-reported sexual orientation } \\
\hline Not sure & $19 / 519$ & 3.7 & $6 / 19$ & 31.6 & $1.5(0.6-4.2)$ \\
\hline Homosexual & $30 / 519$ & 5.8 & $15 / 30$ & 50.0 & $3.4(1.6-7.1)^{* *}$ \\
\hline Bisexual & $13 / 519$ & 2.5 & $4 / 13$ & 30.8 & $1.5(0.5-5.0)$ \\
\hline Heterosexual & $457 / 519$ & 88.0 & $105 / 457$ & 23.0 & 1 \\
\hline \multicolumn{6}{|l|}{ Insurance } \\
\hline Have & $466 / 519$ & 89.8 & $111 / 466$ & 23.8 & $0.6(0.3-1.0)$ \\
\hline No & $53 / 519$ & 10.2 & $19 / 53$ & 35.8 & 1 \\
\hline \multicolumn{6}{|l|}{ HIV Status $^{e}$} \\
\hline Positive & $50 / 519$ & $50 / 519$ & $18 / 50$ & 36.0 & $1.7(0.9-3.2)$ \\
\hline Unknown & $167 / 519$ & $167 / 519$ & $43 / 167$ & 25.7 & $0.8(0.5-1.3)$ \\
\hline Negative & $302 / 519$ & $302 / 519$ & $69 / 302$ & 22.8 & 1 \\
\hline
\end{tabular}


Table 1 Demographic characteristics of the participants and associations with suicidal ideation( $N=519)$ (Continued)

\begin{tabular}{|c|c|c|c|c|c|}
\hline \multirow{2}{*}{$\begin{array}{l}\text { Demographic } \\
\text { Characteristics }\end{array}$} & \multicolumn{2}{|c|}{ Number of participants } & \multicolumn{3}{|c|}{ Had suicidal ideation } \\
\hline & $n / N$ & column(\%) & $n / N$ & $\operatorname{row}(\%)$ & $\mathrm{OR}(95 \% \mathrm{Cl})$ \\
\hline \multicolumn{6}{|l|}{ Type of STls } \\
\hline Gonorrhea & $14 / 519$ & 2.7 & $2 / 14$ & 14.3 & $0.3(0.1-1.5)$ \\
\hline Syphilis & $124 / 519$ & 23.9 & 29/124 & 23.4 & $0.5(0.3-1.1)$ \\
\hline Genital warts & $247 / 519$ & 47.6 & $61 / 247$ & 24.7 & $0.6(0.3-1.1)$ \\
\hline Genital herpes & $12 / 519$ & 2.3 & $3 / 12$ & 25.0 & $0.6(0.1-2.5)$ \\
\hline Others & $72 / 519$ & 13.9 & $17 / 72$ & 23.6 & $0.5(0.2-1.2)$ \\
\hline HIV & $50 / 519$ & 9.6 & $18 / 50$ & 36.0 & 1 \\
\hline \multicolumn{6}{|c|}{ Have had suicidal ideation in life time } \\
\hline Yes & $130 / 519$ & 25.0 & & & \\
\hline No & $389 / 519$ & 75.0 & & & \\
\hline
\end{tabular}

$\overline{a, b, c, d}$ Classification in accordance with China's regulations

eHIV status: unknown including not detected

${ }^{* *} p<0.01$

\section{Psychosocial health conditions}

Of the participants, $24.9 \%$ (129/519) were classified as lonely, $23.7 \%(123 / 519)$ at a high level of entrapment, 24.1\% (125/ $519)$ with defeat, and $22.9 \%(119 / 519)$ as having unmet interpersonal needs. Of the participants, 25.8\% (134/519) lacked social support. About half of the participants (48.4\%; 251/
519) showed depression via their PHQ-9 scores. A total of $38.9 \%(202 / 519)$ had a low level of self-esteem (Table 2).

Demographic characteristics associated with suicidal ideation Table 1 shows the univariable association between participants' demographic characteristics and their suicidal

Table 2 Psychosocial health conditions among participants ( $N=519)$

\begin{tabular}{|c|c|c|c|c|c|}
\hline Psychosocial Health Conditions & $\mathrm{n} / \mathrm{N}$ & column(\%) & Median & Media & SD \\
\hline \multicolumn{6}{|l|}{ Self-esteem $^{a}$} \\
\hline High level (score $\geq 29$ ) & $317 / 519$ & 61.1 & 29.0 & 29.7 & 4.2 \\
\hline Low level $($ score < 29) & $202 / 519$ & 38.9 & & & \\
\hline \multicolumn{6}{|l|}{ Loneliness $^{b}$} \\
\hline High level (score > $\left.P_{75}, 18\right)$ & $129 / 519$ & 24.9 & 14.0 & 14.8 & 5.0 \\
\hline Low level (score $\leq P_{75}, 18$ ) & 390/519 & 75.1 & & & \\
\hline \multicolumn{6}{|l|}{ Depression $^{c}$} \\
\hline Yes (score > 5) & $251 / 519$ & 48.4 & 5.0 & 6.3 & 5.4 \\
\hline No (score $\leq 5$ ) & $268 / 519$ & 51.6 & & & \\
\hline \multicolumn{6}{|l|}{ Entrapment $^{\mathrm{b}}$} \\
\hline High level (score $\left.>P_{75}, 21\right)$ & $123 / 519$ & 23.7 & 8.0 & 12.3 & 13.2 \\
\hline Low level (score $\left.\leq P_{75}, 21\right)$ & $396 / 519$ & 76.3 & & & \\
\hline \multicolumn{6}{|l|}{ Defeat $^{\mathrm{b}}$} \\
\hline High level (score $>P_{75}, 23$ ) & $125 / 519$ & 24.1 & 14.0 & 16.5 & 11.0 \\
\hline Low level $\left(\right.$ score $\left.\leq P_{75}, 23\right)$ & $394 / 519$ & 75.9 & & & \\
\hline \multicolumn{6}{|l|}{ Interpersonal need ${ }^{\mathrm{b}}$} \\
\hline High level $\left(\right.$ score $\left.>P_{75}, 49\right)$ & $119 / 519$ & 22.9 & 34.0 & 36.2 & 14.7 \\
\hline Low level (score $\left.\leq \mathrm{P}_{75}, 49\right)$ & $400 / 519$ & 77.1 & & & \\
\hline \multicolumn{6}{|l|}{ Perceived social support ${ }^{d}$} \\
\hline High level (score > $\left.P_{25}, 59\right)$ & $385 / 519$ & 74.2 & 70.0 & 66.6 & 14.0 \\
\hline Low level (score $\leq P_{25}, 59$ ) & $134 / 519$ & 25.8 & & & \\
\hline
\end{tabular}

acutoff of SES: 29

${ }^{\text {b cutoff: } \mathrm{P}_{75}}$

cutoff: 5

${ }^{d}$ cutoff: $P_{25}$ 
ideation. Four demographic variables (age, marital status, income, and self-reported sexual orientation) showed significant relation to suicidal ideation. Participants aged $<25$ years had more than four times (odds ratio [OR]: 4.7; 95\% confidence interval [CI]: 1.6-13.9) higher reports of suicidal ideation than those aged $\geq 60$ years. Married participants, compared with unmarried, were less likely to have suicidal ideation (OR: 0.5 ; $95 \% \mathrm{CI}$ : $0.3-$ 0.8 . The highest income group was less likely than the lowest to have suicidal ideation (OR: 0.4; 95\%CI: $0.2-$ 0.8). Homosexual respondents were more likely to report suicidal ideation than heterosexual respondents (OR: 3.4; 95\%CI: 1.6-7.1).

\section{Psychosocial problems associated with suicidal ideation}

Table 3 summarizes the binary regression results. After adjusting for age, marital status, income, and selfreported sexual orientation, six of the seven total psychosocial variables constructed among syndemic psychosocial factors showed statistical significance with experiencing suicidal ideation. Participants demonstrating a higher level of loneliness (adjusted odds ratio
[AOR]: 2.4; 95\%CI: 1.5-3.7), depression (AOR: 6.1; 95\%CI: 3.7-10.1), entrapment (AOR: 4.5; 95\%CI: $2.9-$ 7.2), defeat (AOR: 3.5; 95\%CI: 2.2-5.3), unsatisfied interpersonal needs (AOR: 1.9; 95\%CI: 1.2-3.1), or a low level of self-esteem (AOR: 2.1; 95\%CI: 1.4-3.3) were at increased risk for suicidality. Social support was not significantly associated with suicidal ideation among these STI patients. However, the multivariable logistic regression showed that only two psychosocial factors remained significant: entrapment (ORs obtained from forward stepwise multivariable logistic regression [ORm]: 2.1; 95\% CI: $1.1-$ 4.1) and depression (ORm: 4.1; 95\% CI: 2.3-7.2).

\section{Verification of Syndemic effect of psychosocial variables}

Table 4 shows the results of the final syndemic analysis. Generally, it was found that having at least two concurrent psychosocial health problems had a syndemic effect in fusing suicidal ideation (AOR: 4.9; 95\%CI: 3.1-7.8). The low-level group (AOR: 4.2; 95\%CI: 2.6-6.8) and high-level group (AOR: 7.0; 95\%CI: 3.9-12.5) showed a prominent syndemic effect compared with those in the non-syndemic group.

Table 3 Psychosocial problems associated with suicidal ideation among participants $(N=519)$

\begin{tabular}{|c|c|c|c|c|c|}
\hline Psychosocial Problems & $n / N$ & $\operatorname{row}(\%)$ & $\begin{array}{l}\mathrm{OR}^{\mathbf{a}} \\
(95 \% \mathrm{Cl})\end{array}$ & $\begin{array}{l}\text { AOR }^{\mathbf{b}} \\
(95 \% \mathrm{Cl})\end{array}$ & $\begin{array}{l}\mathrm{ORm}^{\mathbf{c}} \\
(95 \% \mathrm{Cl})\end{array}$ \\
\hline \multicolumn{6}{|l|}{ Self-esteem } \\
\hline Low level (score < 29) & $71 / 202$ & 35.1 & $2.4(1.6-3.6)^{* *}$ & $2.1(1.4-3.3)^{* *}$ & \\
\hline High level (score $\geq 29$ ) & $59 / 317$ & 18.6 & 1 & 1 & \\
\hline \multicolumn{6}{|l|}{ Loneliness } \\
\hline High level (score $\left.>P_{75}, 18\right)$ & $52 / 129$ & 40.3 & $2.7(1.8-4.2)^{* *}$ & $2.4(1.5-3.7)^{* *}$ & \\
\hline Low level (score $\left.\leq P_{75}, 18\right)$ & $78 / 390$ & 20.0 & 1 & 1 & \\
\hline \multicolumn{6}{|l|}{ Depression } \\
\hline Yes (score > 5) & $104 / 251$ & 41.4 & $6.6(4.1-10.6)^{* *}$ & $6.1(3.7-10.1)^{* *}$ & $4.1(2.3-7.2)^{* *}$ \\
\hline No (score $\leq 5$ ) & $26 / 268$ & 9.7 & 1 & 1 & \\
\hline \multicolumn{6}{|l|}{ Entrapment } \\
\hline High level (score $\left.>P_{75}, 21\right)$ & $63 / 123$ & 51.2 & $5.2(3.3-8.0)^{* *}$ & $4.5(2.9-7.2)^{* *}$ & $2.1(1.1-4.1)^{*}$ \\
\hline Low level (score $\leq P_{75}, 21$ ) & $67 / 396$ & 16.9 & 1 & 1 & \\
\hline \multicolumn{6}{|l|}{ Defeat } \\
\hline High level (score $\left.>P_{75}, 23\right)$ & $56 / 125$ & 44.8 & $3.5(2.3-5.4)^{* *}$ & $3.5(2.2-5.3)^{* *}$ & \\
\hline Low level (score $\leq P_{75}, 23$ ) & $74 / 394$ & 18.8 & 1 & 1 & \\
\hline \multicolumn{6}{|l|}{ Interpersonal need } \\
\hline High level (score $\left.>P_{75}, 49\right)$ & $42 / 119$ & 35.3 & $1.9(1.2-3.0)^{* *}$ & $1.9(1.2-3.1)^{* *}$ & \\
\hline Low level $\left(\right.$ score $\left.\leq P_{75}, 49\right)$ & $88 / 400$ & 22.0 & 1 & 1 & \\
\hline \multicolumn{6}{|l|}{ Perceived social support } \\
\hline Low level (score $\leq P_{25}, 59$ ) & $39 / 385$ & 10.1 & $1.3(0.9-2.1)$ & $1.3(0.8-2.1)$ & \\
\hline High level (score $\left.>P_{25}, 59\right)$ & $91 / 134$ & 67.9 & 1 & 1 & \\
\hline
\end{tabular}

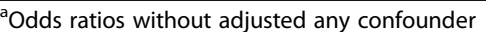

${ }^{b}$ Odds ratios adjusted for significant demographic variables including age, marital status, income, self-reported sexual orientation

'Odds ratios obtained from forward stepwise multivariable logistic regression using significant variables of the univariate analysis as input

${ }^{*} p<0.05$

$*^{*} p<0.01$ 
Table 4 Association between the number of psychosocial problems and suicidal ideation among participants $(\mathrm{N}=519)$

\begin{tabular}{|c|c|c|c|c|c|}
\hline & \multirow[t]{2}{*}{$n / N$} & \multirow[t]{2}{*}{ Column(\%) } & \multicolumn{3}{|c|}{ had suicidal ideation } \\
\hline & & & $n / N$ & $\operatorname{row}(\%)$ & AOR $(95 \% C l)$ \\
\hline \multicolumn{6}{|l|}{ Model 1} \\
\hline \multicolumn{6}{|l|}{ Have a syndemic } \\
\hline Yes & $257 / 519$ & 49.5 & $100 / 257$ & 38.9 & $4.9(3.1-7.8)^{* *}$ \\
\hline No & $262 / 519$ & 50.5 & $30 / 262$ & 11.5 & 1 \\
\hline \multicolumn{6}{|l|}{ Model 2} \\
\hline \multicolumn{6}{|l|}{ Number of psychosocial problems } \\
\hline 5 or more psychosocial problems & $80 / 519$ & 15.4 & $38 / 80$ & 47.5 & $7.0(3.9-12.5)^{* *}$ \\
\hline 2 to 4 psychosocial problems & $177 / 519$ & 34.1 & $62 / 177$ & 35.0 & $4.2(2.6-6.8)^{* *}$ \\
\hline No & $262 / 519$ & 50.5 & $30 / 262$ & 11.5 & 1 \\
\hline
\end{tabular}

\section{Discussion}

The rate of lifetime suicidal ideation among STI patients in Shanghai in our study was 25.0\% (130/519). Among people living with HIV/AIDS in the present study, 18 showed suicidal ideation $(36.0 \% ; 18 / 50)$. In this study, suicidal ideation was independently associated with six psychosocial problems, though not social support. Our most important finding is the syndemic effect of entrapment and defeat in addition to depression, self-esteem, interpersonal needs, loneliness, and social support in suicidal ideation among STI patients, especially in those who had five or more concurrent psychosocial problems.

The difference in suicidal ideation prevalence between our sample (25.0\%) and that of a Canadian sample (6.7\%) [8] could be explained as follows. First, we measured lifetime suicidal ideation and the Canadian research team measured suicidal ideation during the previous 12 months. In addition, the patient demographics in the two studies were substantially different. For example, $65 \%$ of the patients in the Canadian study were in a sexual minority, whereas $88 \%$ of the patients in our study were heterosexual. The suicidal ideation prevalence of PLWHA in our study was $36.0 \%$. This is similar to previously reported prevalences, which range from 27.2 to $43.1 \%$ [9-12]. The significant demographic variables associated with suicidal ideation agreed with findings in other studies [52]. Young participants were at a higher risk of suicidal ideation, possibly owing to higher impulsivity, lower ability to assume responsibility, and lower psychological ability to counteract feelings of vulnerability when faced with harsher social discrimination or mistreatment, which agreed with findings in a study in Swaziland and another in China [27, 53]. Instrumental and emotional support, and financial ability to enable better treatment resources, may explain marital status and higher income as protective factors according to some studies of STI patients in China [54-56]. Research has shown higher suicidality prevalence among male homosexual orientation, in line with the results of our present research $[57,58]$. HIV status was not associated with suicidal ideation, which alerts us that STI patients without HIV may suffer the same high rate of ideation as in HIV patients [59]. Consistent with previous studies, our study found suicidal ideation was independently associated with low self-esteem, depression, loneliness, entrapment, defeat, and interpersonal need. According to cognitive theory, low self-esteem is processed in a typically negative manner, which leads to negative selfappraisal and later to suicidal ideation [60]. As suicidal ideation is among the diagnostic criteria for depression, its presence in and of itself will necessarily increase the number of depressive symptoms. Feeling lonely was associated with distress, which is strongly associated with generalized anxiety, panic attacks, and suicidal ideation [61]. The association between loneliness and suicidality supports the theory that thwarted belongingness and perceived burdensomeness are major determinants of suicidality [31]. The relationship between defeat, entrapment, and suicidal ideation is the motivational phase of the IMV model [30], and also established key variables within Williams' cry of pain theory of suicide [29]. Previous research is equivocal regarding the relationship between social support and suicidal ideation.

When we included all psychosocial variables in a single model, the association with suicidal ideation was removed for most variables, though it remained strongest for depression and entrapment. This should not be surprising, considering suicidal ideation and behavior are known outcomes of feeling trapped in a stressful situation, with no evident escape or rescue [62]; this result has been found in diverse populations and in the context of various disorders and research methodologies [56, 57]. Despite prevalence of poor psychosocial status among STI patients, huge gaps are still visible in concern and service, due to inadequate information and emotional support, and a shortage of qualified professional psychosocial treatment [13, 63-65]. 
To show that suicidal ideation also co-occurs among STI patients, we have extended previous research that confirmed there was a syndemic effect on suicidal ideation in MSM [32]. We confirmed all seven psychosocial health problems tend to co-occur and act to raise risk levels for suicidal ideation in these patients. However, the measurement of the syndemic construct is not invariant across groups; therefore, the construct's meaning also differs. A previous study showed syndemics are in fact a general human phenomenon, but their composition differs, and the consequences are felt most deeply by those in the minority, such as MSM and men who have sex with men and women [36]. These results support the notion that syndemic theory has the potential to advance research, theory, and interventions related to suicidal ideation in this population.

Prevention and intervention efforts should be designed and implemented to reduce suicidal ideation in STI patients. Especially in hospitals, psychological counseling and interventions are needed to improve individuals' mental well-being and reduce suicidal ideation [66-69]. In China, such efforts, especially well-designed psychosocial programs, are still strongly needed for most STI patients and PLWHA [70-73].

\section{Limitations and future research}

Several limitations should be considered in interpreting the present results. First, cross-sectional surveys have difficulty determining causality; therefore, a prospective study assessing recent STI diagnoses and suicidal ideation would be beneficial. Second, although the participants came from a representative hospital, the sample size was not especially large; multi-center research is needed. Third, although privacy was ensured, investigators were trained, and doctors' cooperation ensured survey quality, self-reported and recall bias were unavoidable. Fourth, our findings do not indicate the incidence of suicidal ideation in newly diagnosed and revisited patients; future studies are needed to focus on and explore the differences between the two. Fifth, there was selection bias. For example, we excluded from this study neurosyphilis patients with severe mental or cognitive impairment; patients who had experienced associated stigma may also have been more reluctant to participate. And accidental sampling reduces the possibility of replicability and generalization. Sixth, we used a self-reported binary scale assess lifetime suicidal ideation, which potentially underestimated its prevalence. Finally, the syndemic effect examined classification variables using a cutoff point, which may not supply an adequate amount of information, although the results for continuous variables were similar.

\section{Conclusions}

This study adds to the literature in several important ways. We demonstrated that not only PLWHA but also other STI patients have high rates of suicidal ideation, and this population suffers from severe psychosocial problems. This study identified a syndemic effect of psychosocial problems on increasing odds of suicidal ideation. The collective findings suggest that greater attention should be paid to STI patients' psychosocial well-being; especially in hospitals. Efforts to prevent suicidal ideation, as well as other mental problems, among STI patients are therefore urgently needed to improve the social and health conditions for this population.

\section{Abbreviations \\ STD: Sexually transmitted disease; STI: Sexually transmitted infection; HIV: Human immunodeficiency virus; AIDS: Acquired Immune deficiency syndrome; PLWHA: People living with HIV/AIDSIMV model: integrated motivational-volitional model; IPTS: Interpersonal theory of suicide; MSM: Men who have sex with men; SES: Rosenberg Self-esteem Scale; ULS- 8: Eight-item UCLA Loneliness Scale; PHQ-9: Nine-item Patient Health Questionnaire; ES: 16-item Entrapment Scale; DS: 16-item Defeat Scale; INQ- 15: 15-item Interpersonal Need Questionnaire; MSPSS: Multidimensional Scale of Perceived Social Support; OR: Odd ratio; Cl: Confidence interval; AOR: Adjusted odd ratio; ORm: Odds ratios obtained from forward stepwise multivariable logistic regression}

\section{Acknowledgements}

The work involved in collection of questionnaires was completed in collaboration with Dr. Pingyu Zhou and Yang Ni at the Shanghai Skin Disease Hospital. We thank Liwen Bianji, Edanz Editing China (www. liwenbianji.cn/ac), for editing a draft of this manuscript.

\section{Authors' contributions}

YC substantially contributed to the conceptualization and design of this research, and to revising the manuscript. JM contributed to conceptualizing this manuscript and also wrote sections of the manuscript. SW assisted the study design, data collection, and registration of the data from hospital files, data analyses, data interpretation, and drafting the manuscript. RG contributed to data analyses, data interpretation, and to drafting and revising the manuscript. YS made great efforts to recruit eligible participants and to collect data. YN substantially contributed to the data collection and cooperation with the hospital. All authors read and approved the final manuscript.

\section{Funding}

National Natural Science Foundation of China, 71673187—Research on intervention strategies for HIV high-risk behaviors of transgender men with male behaviors based on IMB model. The funders had no role in the design of the study; in the collection, analysis, and interpretation of data; or in writing the manuscript.

\section{Availability of data and materials}

The datasets used and/or analysed during the current study are available from the corresponding author on reasonable request.

\section{Ethics approval and consent to participate}

The Shanghai Jiao Tong University School of Medicine Public Health and Nursing Ethics Committee approved the present study (approval number: SJUPN-201702). Informed, written consent was obtained from all individual participants included in the study.

Consent for publication

Not applicable.

Competing interests

The authors declare they have no competing interests. 


\section{Author details}

'School of Public Health, Shanghai Jiao Tong University School of Medicine, No. 227 South Chongqing Road, Shanghai 200025, China. ${ }^{2}$ Shanghai Skin Disease Hospital, Tongji University School of Medicine, No. 1278 Baode Road, Shanghai 200443, China. ${ }^{3}$ Shanghai Xuhui Center for Disease Control and Prevention, No. 50 Yongchuan Road, Shanghai 200237, China.

\section{Received: 4 June 2019 Accepted: 18 August 2020}

\section{Published online: 31 August 2020}

\section{References}

1. Centers for Disease Control and Prevention. Definitions: self-directed violence. Atlanta: CDC; 2014

2. Chen Y, Wu J, Yi Q, Huang G, Wong T. Depression associated with sexually transmitted infection in Canada. Sex Transm Infect. 2008;84(7):535-40. https://doi.org/10.1136/sti.2007.029306.

3. Ginieri-Coccossis M, Triantafillou E, Papanikolaou N, et al. Quality of life and depression in chronic sexually transmitted infections in UK and Greece: The use of WHOQOL-HIV/STI BREF. Psychiatriki. 2018;29(3):209-19. https://doi. org/10.22365/jpsych.2018.293.209..

4. Jenkins WD, Botchway A. Young adults with depression are at increased risk of sexually transmitted disease. Prev Med. 2016;88:86-9. https://doi.org/10. 1016/j.ypmed.2016.03.020.

5. Koegler E, Kennedy CE, Thompson CB, Winch PJ, Mpanano RM, Perrin NA, Glass N. Association between self-reported sexually transmitted infection treatment and mental health symptoms in conflict-affected eastern Democratic Republic of the Congo. Int J STD AIDS. 2071;29(3):212-20. https://doi.org/10.1177/0956462417722128..

6. Agardh A, Cantor-Graae E, Ostergren PO. Youth, sexual risk-taking behavior, and mental health: a study of university students in Uganda. Int J Behav Med. 2012;19(2):208-16. https://doi.org/10.1007/s12529-011-9159-4.

7. Huang SY, Hung JH, Hu LY, Huang MW, Lee SC, Shen CC. Risk of sexually transmitted infections following depressive disorder: a nationwide population-based cohort study. Medicine (Baltimore). 2018;97(43):e12539. https://doi.org/10.1097/MD.0000000000012539.

8. Salway T, Ferlatte O, Shoveller J, et al. The Need and Desire for Mental Health and Substance Use-Related Services Among Clients of PubliclyFunded Sexually Transmitted Infection Clinics in Vancouver, Canada. J Public Health Manag Pract. 2019;25(3):E1-10.https://doi.org/10.1097/PHH. 0000000000000904

9. Lau JTF, Yu XN, Mak WWS, et al. Suicidal ideation among HIV+ former blood and/or plasma donors in rural China. AIDS Care. 2010;22(8):9.

10. Atkinson $\mathrm{JH}$, Duarte NA, Franklin D, et al. Risks and predictors of current Suicidality in HIV-infected heroin users in treatment in Yunnan, China: a controlled study. J Acquir Immune DeficSyndr. 2013;62(3):311-6.

11. Huiying W, Min W, Furong J, et al. Socio-psychological factors relevant to suicidal ideation among patients with AIDS in Changsha. Zhong Nan Da Xue Xue Bao Yi Xue Ban. 2017:42(6):687-94 In Chinese.

12. Badiee J, Moore DJ, Atkinson $\mathrm{JH}$, et al. Lifetime suicidal ideation and attempt are common among HIV+ individuals. J Affect Disord. 2012;136(3): 993-9.

13. Rebar AL, Vandelanotte C, Van Uffelen J, et al. Associations of overall sitting time and sitting time in different contexts with depression, anxiety, and stress symptoms. Ment Health Phys Act. 2014:7(2):105-10.

14. Ahmad OB, Boschi-Pinto C, Lopez AD, Murray CJL, Lozano R, et al. Age standardization of rates: A new WHO standard. GPEDiscussion Paper No. 31. Geneva: Global Programme on Evidence for Health Policy, World Health Organization; 2000. Available: http://w3.whosea.org/healthreport/pdf/ paper31.pdf. Accessed 25 Mar 2005

15. Mruk C. Self-esteem: research, theory, and practice: towards a positive psychology of self-esteem. New York: Springer Publishing; 1995.

16. Bryan CJ, Hernandez AM. The functions of social support as protective factors for suicidal ideation in a sample of air force personnel. Suicide Life Threat Behav. 2013;43(5):562-73.

17. Chatard A, Selimbegovic L, Konan PND. Self-esteem and suicide rates in 55 nations. Eur J Personal. 2009;23(1):19-32.

18. de Man AF, Gutierrez BIB. The relationship between level of self-esteem and suicidal ideation with stability of self-esteem as moderator. Can J Behav Sci. 2002:34(4):235-8
19. Kleiman EM, Riskind JH. Utilized social support and self-esteem mediate the relationship between perceived social support and suicide ideation. A test of a multiple mediator model crisis. Crisis. 2013;34(1):42-9.

20. Palmer CJ. Suicide attempt history, self-esteem, and suicide risk in a sample of 116 depressed voluntary inpatients. Psychol Rep. 2004;95(3):1092-4.

21. Sharaf AY, Thompson EA, Walsh E. Protective effects of self-esteem and family support on suicide risk behaviors among at-risk adolescents. J Child Adolesc Psychiatr Nurs. 2009;22(3):160-8.

22. Zhang J, Grabiner VE, Zhou Y, Li N. Suicidal ideation and its correlates in prisoners. A comparative study in China. Crisis. 2010;31(6):335-42.

23. Cantwell J, Muldoon O, Gallagher S. The influence of self-esteem and social support on the relationship between stigma and depressive symptomology in parents caring for children with intellectual disabilities. J Intellect Disabil Res. 2015;59(10):10.

24. Blankstein KR, Lumley $\mathrm{CH}$, Crawford A. Perfectionism, hopelessness, and suicide ideation: revisions to diathesis-stress and specific vulnerability models. J Rational Emotive Cogn Behav Ther. 2007:25:279-319.

25. Lieberman Z, Solomon Z, Ginzburg K. Suicidal ideation among young adults: effects of perceived social support, self-esteem, and adjustment. Loss Trauma. 2005;10:163-81.

26. Wilke DJ. Predicting suicide ideation for substance users: the role of selfesteem, abstinence, and attendance at 12-step meetings. Addict Res Theory. 2004;12:231-40.

27. Almansour AM, Seter S. Suicidal ideation and associated factors among school going adolescents in Swaziland. African Health Sci. 2018;17(4): 1172

28. Teo AR, Marsh HE, Forsberg CW, et al. Loneliness is closely associated with depression outcomes and suicidal ideation among military veterans in primary care. J Affect Disord. 2018;230:42-9. https://doi.org/10.1016/j.jad. 2018.01 .003

29. Williams JMG, Pollock LR. Psychological aspects of the suicidal process. Underst Suicidal Behav. 2001:76-93.

30. O'Connor RC, Cleare S, Eschle S, Wetherall K, Kirtley OJ. The integrated motivational-volitional model of suicidal behavior. In: O'Connor RC, Pirkis J, editors. The international handbook of suicide prevention. Chichester: Wiley; 2016. p. 220-40.

31. Joiner T. Why people die by suicide. Amwa J Am Med Writers Assoc J. 2005 3:2082-3.

32. Li R, Cai Y, Wang Y, et al. Psychosocial Syndemic associated with increased suicidal ideation among men who have sex with men in Shanghai, China. Health Psychology Official J Division Health Psychol Am Psycholog Assoc. 2015;35(2):148.

33. Wang W, Xiao C, Yao X, Yang Y, Yan H, Li S. Psychosocial health and suicidal ideation among people living with HIV/AIDS: A crosssectional study in Nanjing, China. PLoS One. 2018;13(2):e0192940. Published 2018 Feb 22. https://doi.org/10.1371/journal.pone.0192940.

34. Tsai AC. Syndemics: A theory in search of data or data in search of a theory? Soc Sci Med. 2018;206:117-22. https://doi.org/10.1016/j.socscimed. 2018.03.040

35. Bantjes J, Kagee A, Saal W. Suicidal ideation and behaviour among persons seeking HIV testing in peri-urban areas of Cape Town, South Africa: a lost opportunity for suicide prevention. AIDS Care. 2016:1-9.

36. Mustanski B, Andrews R, Herrick A, et al. A Syndemic of psychosocial health disparities and associations with risk for attempting suicide among young sexual minority men. Am J Public Health. 2014;104(2):287-94.

37. Petersen, W. Society and the Adolescent Self-Image. Morris Rosenberg. Princeton University Press, Princeton, N.J. 1965; 148(3671):804.

38. Wang XD. Manual of Mental Health Assessment Scale (Updated Edition) Beijing: China Journal of Mental Health; 1999. p. 318-20. In Chinese.

39. Russell D, Peplau LA, Ferguson ML. Developing a measure of loneliness. J Pers Assess. 1978:42(3):290-4.

40. Hays RD, Dimatteo MR. A short-form measure of loneliness. J Pers Assess. 1987;51(1):69-81.

41. Liang ZHOU, Zhi LI, Mi HU, Shuiyuan XIAO. Reliability and validity of ULS-8 loneliness scale in elderly samples in a rural community. J Central South Univ (Medical Science). 2012;37(11):1124-8 In Chinese.

42. Baoquan MIN, Aihong ZHOU, Feng LIANG, et al. Clinical application of patient health questionnaire for self-administered measurement (PHQ-9) as screening tool for depression. J Neurosci Mental Health. 2013;13(6):569-72 In Chinese. 
43. Manea L, Gilbody S, Mcmillan D. A diagnostic meta-analysis of the patient health Questionnaire-9 (PHQ-9) algorithm scoring method as a screen for depression. Gen Hosp Psychiatry. 2015;37(1):67-75.

44. Gilbert P, Allan S. The role of defeat and entrapment (arrested flight) in depression: an exploration of an evolutionary view. Psychol Med. 1998;28(3): 585-98.

45. Ruijie GONG, Jingyi LIU, Yichen WANG, Yong CAI, Suping WANG. Validity and reliability of the Chinese vision of the entrapment scale in medical students. Chin Ment Health J. 2019;33(5):393-7 In Chinese.

46. Hua TANG, Su-ping WANG, Rui-jie GONG, Ze-zhou WANG, Yong CAl. Reliability and validity of defeat scale on anxiety and depression in medical students. J Shanghai Jiaotong Univ (Medical Science). 2019;39(1):84-8 In Chinese.

47. Van Orden KA, Cukrowicz KC, Witte TK, et al. Thwarted belongingness and perceived burdensomeness: construct validity and psychometric properties of the interpersonal needs questionnaire [J]. Psychol Assess. 2012;24(1):197215.

48. Li XM, Xin TG, Yuan J, et al. Validity and reliability of the interpersonal needs questionnaire in Chinese college students. Chin J Clin Psychol. 2015;23(4): 590-3. In Chinese.

49. Jarrett SB, Haye WDL, Miller Z, et al. High prevalence of psychiatric and substance use disorders among persons seeking treatment for HIV and other STIs in Jamaica: a short report. AIDS Care. 2018;30(5):604-8.

50. Zhou K, Li H, Wei X, et al. Reliability and validity of the multidimensional scale of perceived social support in Chinese mainland patients with methadone maintenance treatment [J]. Compr Psychiatry. 2015;60:182-8.

51. Hong-Yan WU, Ye-Huan S, Xiu-Jun Z, et al. Study on the social psychology influencing factors of suicidal ideation in people living with AIDS. Chin J Dis Control Prevent. 2007.

52. Yang YF, Luo ZZ, Tan Y, et al. Quality of life and its influencing factors of syphilis patients in Nanshan District of Shenzhen in 2015. [J]. Practical Preventive Medicine. 2016;23(10):1184-7. In Chinese.

53. Li GY. Suicide and Self-inflicted Injury: People's Health Publishing House; 2009. p. 1-73. In Chinese

54. Liao J, Xiong JH, Shi QK, et al. Survey of social support status of HIV/AIDS patients and their families. U.S. Chinese Health Hygiene J. 2003;(2):16-8 In Chinese.

55. Wu D, Wu Y, Zhang XD, et al. Quality of life in syphilis patients. Chinese J Dermatovenereol. 2010;24(12):1118-20 In Chinese.

56. Gui CP, Liu HE, Meng CP. Qualitative Study on Psychological Feelings of Spouses of Syphilis Patients. Shanxi Med J (second half monthly). 2011;40(6): 292-3 In Chinese.

57. Zhang BC, XF LI, QS Chu, et al. Survey on AIDS-related status of 2046 gay men in nine cities of China. Chinese J Human Sexual. 2008;17(8):6-10 In Chinese.

58. Zhang $\mathrm{XH}$, Zeng $\mathrm{CB}$, Cai WO, et al. Gender differences in suicide status and influencing factors among HIV/AIDS patients. Chinese J AIDS STD. 2016;8: 601-4 In Chinese.

59. YC X. Investigation on psychological status of STD patients and nursing intervention. China Med Herald. 2010;07(30):130-1 In Chinese.

60. Clark DM, Salkovskis PM, Hackmann A, et al. Brief cognitive therapy for panic disorder: a randomized controlled trial. J Consult Clin Psychol. 1999;67(4): 583-9.

61. Beutel ME, Klein EM, Brahler $\mathrm{E}$, et al. Loneliness in the general population: prevalence, determinants and relations to mental health. BMC Psychiatry. 2017;17(1):97.

62. Williams PD, Pollock DD, Goldstein RA. Evolution of functionality in lattice proteins. J Mol Graphics Modelling. 2001;19(1):150-6.

63. Gao X, Jackson $\mathrm{T}$, Chen $\mathrm{H}$, et al. There is a long way to go: a nationwide survey of professional training for mental health practitioners in China. Health Policy. 2010;95(1):0-81..

64. Jacob KS, Sharan P, Mirza I, et al. Mental health systems in countries: where are we now? Lancet. 2007;370(9592):0-1077.

65. Qian J. Mental health care in China: providing services for under-treated patients. Journal of Mental Health Policy Economics. 2012;15(4):179.

66. Hasanah Cl, Zaliha AR, Mahiran M. Factors influencing the quality of life in patients with HIV in Malaysia. Qual Life Res. 2011;20(1):91-100..

67. Brown $J$, Vanable PA. Cognitive-behavioral stress management interventions for persons living with HIV: a review and critique of the literature. Ann Behav Med. 2008;35(1):26-40.
68. Himelhoch S, Medoff DR, Oyeniyi G. Efficacy of group psychotherapy to reduce depressive symptoms among HIV-infected individuals: a systematic review and meta-analysis. AIDS Patient Care STDs. 2007;21(10):732-9.

69. Scott-Sheldon LA, Kalichman SC, Carey MP, Fielder RL. Stress management interventions for HIV+ adults: a meta-analysis of randomized controlled trials, 1989 to 2006. Health Psychol. 2008;27(2):129-39.

70. Sullivan SG, Xu J, Feng Y, Su S, Xu C, Ding X, Gao Y, Dou Z, Wu Z, et al. Stigmatizing attitudes and behavior towards PLHA in rural China. AIDS Care. 2010;22(1):104-11.

71. Albrektsson M, Alm L, Tan X, Andersson R. HIV/AIDS awareness, attitudes and risk behavior among university students in Wuhan, China. Open AIDS J. 2009;3:55-62.

72. Kraaij V, van Emmerik A, Garnefski N, Schroevers MJ, Lo-Fo-Wong D, van Empelen PV, Dusseldorp E, Witlox R, Maes S. Effects of a cognitive behavioral self-help program and a computerized structured writing intervention on depressed mood for HIV-infected people: a pilot randomized controlled trial. Patient Educ Couns. 2010;80(2):200-4.

73. Zeng C, Li L, Hong YA, et al. A structural equation model of perceived and internalized stigma, depression, and suicidal status among people living with HIV/AIDS [J]. BMC Public Health. 2018;18(1):138.

\section{Publisher's Note}

Springer Nature remains neutral with regard to jurisdictional claims in published maps and institutional affiliations.
Ready to submit your research? Choose BMC and benefit from:

- fast, convenient online submission

- thorough peer review by experienced researchers in your field

- rapid publication on acceptance

- support for research data, including large and complex data types

- gold Open Access which fosters wider collaboration and increased citations

- maximum visibility for your research: over $100 \mathrm{M}$ website views per year

At BMC, research is always in progress.

Learn more biomedcentral.com/submissions 